\title{
Carbon ionization at gigabar pressures: An ab initio perspective on astrophysical high-density plasmas
}

\author{
Mandy Bethkenhagen $\odot,{ }^{1,2}$ Bastian B. L. Witte, ${ }^{1,3}$ Maximilian Schörner, ${ }^{1,3}$ Gerd Röpke, ${ }^{1}$ Tilo Döppner, ${ }^{4}$ Dominik Kraus, ${ }^{5,6}$ \\ Siegfried H. Glenzer, ${ }^{3}$ Philip A. Sterne, ${ }^{4}$ and Ronald Redmer ${ }^{1}$ \\ ${ }^{1}$ Institut für Physik, Universität Rostock, 18051 Rostock, Germany \\ ${ }^{2}$ CNRS, École Normale Supérieure de Lyon, Laboratoire de Géologie de Lyon LGLTPE UMR 5276, Centre Blaise Pascal, \\ 46 allée d'Italie Lyon 69364, France \\ ${ }^{3}$ SLAC National Accelerator Laboratory, 2575 Sand Hill Road, MS 72, Menlo Park, California 94025, USA \\ ${ }^{4}$ Lawrence Livermore National Laboratory, Livermore, California 94550, USA \\ ${ }^{5}$ Helmholtz-Zentrum Dresden-Rossendorf, 01328 Dresden, Germany \\ ${ }^{6}$ Institute of Solid State and Materials Physics, Technische Universität Dresden, 01069 Dresden, Germany
}

(Received 7 December 2019; accepted 28 April 2020; published 1 June 2020)

\begin{abstract}
A realistic description of partially ionized matter in extreme thermodynamic states is critical to model the interior and evolution of the multiplicity of high-density astrophysical objects. Current predictions of its essential property, the ionization degree, rely widely on analytical approximations that have been challenged recently by a series of experiments. Here, we propose an ab initio approach to calculate the ionization degree directly from the dynamic electrical conductivity using the Thomas-Reiche-Kuhn sum rule. This density functional theory framework captures genuinely the condensed-matter nature and quantum effects typical for strongly correlated plasmas. We demonstrate this capability for carbon and hydrocarbon, which most notably serve as ablator materials in inertial confinement fusion experiments aiming at recreating stellar conditions. We find a significantly higher carbon ionization degree than predicted by commonly used models, yet validating the qualitative behavior of the average atom model PURGATORIO. Additionally, we find the carbon ionization state to remain unchanged in the environment of fully ionized hydrogen. Our results will not only serve as benchmark for traditional models, but more importantly provide an experimentally accessible quantity in the form of the electrical conductivity.
\end{abstract}

DOI: 10.1103/PhysRevResearch.2.023260

\section{INTRODUCTION}

Modeling the internal structure and thermal evolution of low-mass stars, brown dwarfs, and massive giant planets requires accurate equation-of-state data and even more importantly reliable transport properties of warm dense matter $[1,2]$. For example, the interplay of convective and radiative transport in low-mass stars is reflected by key plasma quantities such as opacity, electrical conductivity, and absorption coefficients. All those properties can be directly linked to the ionization degree, which is defined as the ratio between the number of free electrons and the sum of all electrons.

The ionization degree can be obtained directly from the Saha equations for the limiting case of the low-density plasma in thermodynamic equilibrium. In this framework, the corresponding ionization energies are defined as the difference between the ground-state energy and its continuum of free states. Generally, the ionization energies crucially depend on

Published by the American Physical Society under the terms of the Creative Commons Attribution 4.0 International license. Further distribution of this work must maintain attribution to the author(s) and the published article's title, journal citation, and DOI. the temperature and density of a plasma. For example, an increase of the density results in a lowering of the ionization energies with respect to their well-known values for isolated atoms due to correlation effects such as screening of the Coulomb interaction, self-energy, strong ion-ion interactions, and Pauli blocking [3,4]. This effect is known as ionization potential depression (IPD) and is inherent to any theory aiming at predicting the ionization degree, which has been subject of many-particle physics for decades [4-7]. For instance, the simple Debye-Hückel theory for static screening has been combined with the ion sphere model by Ecker and Kröll (EK) [8] and later improved by Stewart and Pyatt (SP) [9]. The predictions of both models differ considerably for high-density plasmas as encountered in the deep interior of astrophysical objects, which are characterized by pressures up to the gigabar range and temperatures of several $\mathrm{eV}$ to $\mathrm{keV}$.

Matter under such extreme conditions is notoriously challenging to produce and probe. However, great advances in $\mathrm{x}$-ray techniques have been made over the last decade and have been implemented at high-power laser facilities and free electron lasers (FELs), which are now available for the experimental study of high-density plasmas. For instance, the ionization state of isochorically heated solid aluminum was extracted at temperatures in the range $10-100 \mathrm{eV}$ by measuring the $K$ edge threshold at the Linac Coherent Light Source 
(LCLS) [10-12]. Additionally, the ionization of hot dense aluminum plasmas in the range $1-10 \mathrm{~g} / \mathrm{cm}^{3}$ and $500-700 \mathrm{eV}$ was determined using the ORION laser [13]. Experiments performed at the Omega laser facility and National Ignition Facility compressed hydrocarbon $(\mathrm{CH})$ up to $100 \mathrm{Mbar}$, and obtained the ionization state via $\mathrm{x}$-ray Thomson scattering (XRTS) $[14,15]$. The same technique was applied at the LCLS to measure the IPD in carbon plasma [16]. Furthermore, the total intensity of plasma emission in $\mathrm{Al}$ and $\mathrm{Fe}$ driven by narrow-bandwidth $\mathrm{x}$-ray pulses across a range of wavelengths was utilized to determine the IPD [17]. Generally, the results of those experiments indicate that rather simple models including IPD based on the EK or SP approaches fail to describe the ionization degree correctly [7].

Therefore, novel theoretical concepts for the prediction of the ionization degree are imperatively required and first improvements have been made. For example, a two-step Hartree-Fock-Slater (HFS) approach has been proposed recently [18]. It is a combined atomic-solid-plasma model that permits ionization potential depression studies also for single and multiple core hole states [19], or the dynamic ion-ion structure factor [20]. The latter approach has been generalized to include Pauli blocking effects which are important at high densities [21]. Another route is to characterize ionization by applying molecular dynamics simulations for the ions in combination with electronic structure calculations using density functional theory (DFT-MD) [22-24], which is especially well suited for dense plasmas. So far, all DFT-MD works relied widely on the density of states (DOS), which was used to analyze the evolution of the ionization degree with density and temperature [22-24]. However, none of these works provided a consistent picture of the ionization degree resolving the recent discussion on IPD in high-density plasmas (see Refs. [21,23,25], the comment of Iglesias and Sterne [26], and the reply by Hu [27]). This debate is fundamentally related to the question of how to define the ionization degree properly for warm dense matter, which is characterized by densities beyond the applicability range of the Saha equations and its underlying chemical picture.

In this work, we meet this challenge by calculating the ionization degree directly from an experimentally accessible quantity: the dynamic electrical conductivity. This DFT-MD method takes into account the electronic and ionic correlations in a self-consistent way and, in particular, reflects essential features of high-density plasmas such as the existence of energy bands instead of sharp atomic levels and their occupation according to Fermi-Dirac statistics. In contrast to a definition relying entirely on the DOS, our approach is based on the Thomas-Reiche-Kuhn (TRK) sum rule and the evaluation of electronic transitions originating solely from electrons within the conduction band. This method, which has, to our knowledge, never been used before, is a step toward precisely modeling matter at high energy densities as occurring, e.g., in inertially confined fusion experiments [28] or in low-mass stars [1], brown dwarfs, and massive giant planets [2]. Carbon and $\mathrm{CH}$ are chosen as exemplary materials relevant to the afore-mentioned applications.

\section{METHODS}

\section{A. Deriving ionization from the dynamic conductivity and the sum rule}

The dynamic electrical conductivity, also referred to as optical conductivity, is calculated from the Kubo-Greenwood formula,

$$
\begin{aligned}
\sigma^{\mathrm{tot}}(\omega)= & \frac{2 \pi e^{2}}{3 V \omega} \sum_{\mathbf{k} v \mu}\left(f_{\mathbf{k} v}-f_{\mathbf{k} \mu}\right)|\langle\mathbf{k} v|\hat{\mathbf{v}}| \mathbf{k} \mu\rangle|^{2} \\
& \times \delta\left(E_{\mathbf{k} \mu}-E_{\mathbf{k} v}-\hbar \omega\right),
\end{aligned}
$$

which can be derived from linear response theory [29-32]. In the above equation, the transition matrix elements $|\langle\mathbf{k} v|\hat{\mathbf{v}}| \mathbf{k} \mu\rangle|^{2}$ with the velocity operator $\hat{\mathbf{v}}$ are the key components. They reflect the transition probability between the initial eigenstate associated with band $v$ and the final eigenstate in band $\mu$ at a particular $\mathbf{k}$ point in the Brillouin zone of the simulation box of volume $V$. For a given frequency $\omega$, only states with a positive difference between eigenenergies $E_{\mathbf{k} \mu}$ and $E_{\mathbf{k} \nu}$ contribute to the conductivity. The occupation of initial and final states is weighted with the Fermi-Dirac function $f_{\mathbf{k} v}=\left[\exp \left(\left(E_{\mathbf{k} v}-\mu_{\mathrm{e}}\right) / k_{B} T\right)+1\right]^{-1}$, whereas $T$ and $\mu_{\mathrm{e}}$ denote the temperature and the chemical potential of the electrons, respectively. Additionally, $e$ and $\hbar$ represent the elementary charge and the reduced Planck constant in Eq. (1).

The resulting dynamical electrical conductivity has to fulfill the well-known TRK sum rule for dipole transitions [33-37],

$$
Z^{\mathrm{tot}}=\frac{N_{\mathrm{e}}^{\mathrm{tot}}}{N_{\mathrm{i}}}=\frac{2 m_{e} V}{\pi e^{2} N_{\mathrm{i}}} \int_{0}^{\infty} d \omega \sigma^{\mathrm{tot}}(\omega) .
$$

It yields the ratio between the total number of electrons, $N_{\mathrm{e}}^{\text {tot }}$, and the number of ionic centers in the system, $N_{\mathrm{i}}$, and establishes an important convergence criterion for the numerical computation of the dynamic electrical conductivity. For the examples chosen in this work, we require charge state values of $Z^{\text {tot }}=6$ for carbon and $Z^{\text {tot }}=7$ for $\mathrm{CH}$ in order to fulfill the TRK sum rule exactly. The number of ionic centers is in both cases $N_{\mathrm{i}}=N_{\mathrm{C}}=N_{\mathrm{CH}}=32$.

Our approach separates the dynamic electrical conductivity $\sigma^{\text {tot }}$ into three individual parts based on the different nature of electronic transitions in the energy spectrum. Hence, the transition matrix elements for a given $\mathbf{k}$ point of the sum in Eq. (1) are divided into contributions attributed to intraband transitions in the conduction (c-c) and valence (v-v) bands as well as interband transitions between the valence and conduction bands $(\mathrm{v}-\mathrm{c})$ :

$$
\sigma^{\text {tot }}(\omega)=\sigma^{\mathrm{c}-\mathrm{c}}(\omega)+\sigma^{\mathrm{v}-\mathrm{c}}(\omega)+\sigma^{\mathrm{v}-\mathrm{v}}(\omega),
$$

whereas each contribution $\mathrm{x}=\{\mathrm{c}-\mathrm{c}, \mathrm{v}-\mathrm{c}, \mathrm{v}-\mathrm{v}\}$ is required to fulfill the partial TRK sum rule,

$$
Z^{\mathrm{x}} \equiv \frac{2 m_{e} V}{\pi e^{2} N_{\mathrm{i}}} \int_{0}^{\infty} d \omega \sigma^{\mathrm{x}}(\omega) .
$$

The individual conductivity contributions can be simply identified by choosing an energy within the energy gap between the valence and conduction bands, which is chosen naturally 
as the center of the gap between the states of interest. For our carbon and $\mathrm{CH}$ examples, the gap is always chosen between the clearly identifiable $1 s$ valence and the continuum as conduction states, which already comprise the $2 s$ and $2 p$ states at the considered conditions.

The electrons effectively contributing to the conductivity in the conduction band are the free electrons $N_{e}^{\text {free }}$, so that we can identify $\sigma^{\mathrm{x}}(\omega)=\sigma^{\mathrm{c}-\mathrm{c}}(\omega)$ in Eq. (4) and define the ionization state as

$$
Z^{\text {free }}=\frac{N_{\mathrm{e}}^{\text {free }}}{N_{\mathrm{i}}} \equiv Z^{\mathrm{c}-\mathrm{c}},
$$

which we propose as a new and suitable measure for this quantity in high-density plasmas.

Finally, we calculate the ionization degree,

$$
\alpha=\frac{Z^{\text {free }}}{Z^{\text {tot }}}=\frac{N_{\mathrm{e}}^{\text {free }}}{N_{\mathrm{e}}^{\text {tot }}},
$$

which is consequently defined as the ratio between the number of free and total charge carriers per number of ionic centers, $N_{\mathrm{i}}$.

\section{B. Computational details}

The DFT-MD simulations for carbon and $\mathrm{CH}$ were performed with the Vienna Ab initio Simulation Package (VASP) [38-40]. We considered densities between 20 and $400 \mathrm{~g} / \mathrm{cm}^{3}$ at a temperature of $100 \mathrm{eV}$ resulting in a pressure range between 0.8 and 65 Gbar. These conditions correspond to a maximum compression ratio of more than 100 and, thus, it was crucial to treat all electrons explicitly using the Coulomb potential with a cutoff energy of $15 \mathrm{keV}$. We considered 32 carbon atoms up to $150 \mathrm{~g} / \mathrm{cm}^{3}$ and 64 carbon atoms for the three highest densities starting at $200 \mathrm{~g} / \mathrm{cm}^{3}$. For the $\mathrm{CH}$ calculations, we added 32 and 64 hydrogen atoms to the respective pure carbon simulations. Additionally, a large number of bands, i.e., typically $800-5000$ bands, was required to describe the 192 electrons for carbon and respectively the 224 electrons for $\mathrm{CH}$ adequately at the high temperature considered here. Each DFT-MD simulation was run for at least 20000 time steps with a step size of 50 as for carbon and 10 as for $\mathrm{CH}$ in order to reflect the ion dynamics properly. A NoséHoover thermostat [41] was used to control the ion temperature, the Brillouin zone was evaluated at the Baldereschi mean value point [42], and we employed the exchange-correlation (XC) functional of Perdew, Burke, and Ernzerhof (PBE) [43].

The electrical conductivity was determined from an average over 20 snapshots taken from the DFT-MD simulation per condition, and a Monkhorst-Pack $2 \times 2 \times 2$ grid was used to evaluate the Kubo-Greenwood formula, Eq. (1). We performed extensive convergence tests of the DFT-MD simulations with respect to the energy cutoff, number of bands, $\mathbf{k}$ point sets, and the number of atoms. Furthermore, we tested the influence of the XC functional used in the DFT cycles by carrying out additional calculations with the local density approximation (LDA) and strongly constrained and appropriately normed (SCAN) [44] functionals. All parameters were chosen such that the TRK sum rule is always fulfilled within $2 \%$, which depends most significantly on the number of explicitly considered bands.

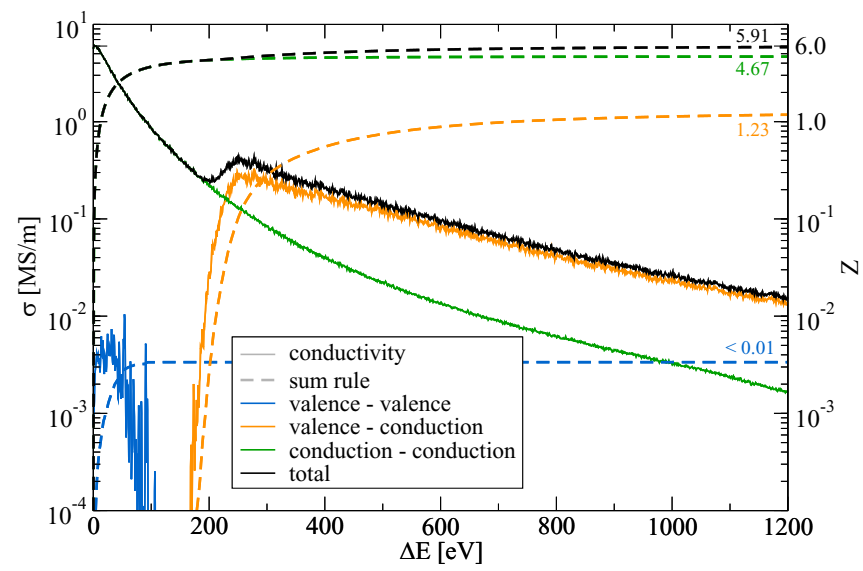

FIG. 1. Electrical conductivity (solid lines) and TRK sum rule values (dashed lines) for a carbon simulation snapshot at $50 \mathrm{~g} / \mathrm{cm}^{3}$ and $T=100 \mathrm{eV}$. The different colors indicate the total value and the individual components of both quantities according to Eqs. (3) and (4). The final TRK sum rule values are given as colored numbers.

\section{RESULTS}

\section{A. Dynamic electrical conductivity and sum rule}

In the following, we demonstrate our method for an exemplary single snapshot of a carbon plasma at $50 \mathrm{~g} / \mathrm{cm}^{3}$ and $100 \mathrm{eV}$. In Fig. 1, the total dynamic electrical conductivity obtained with the Kubo-Greenwood approach is shown as a solid black line. The curve spans three orders of magnitude over the entire considered energy range and exhibits a pronounced local maximum at about $250 \mathrm{eV}$, which results from the strong $\mathrm{v}-\mathrm{c}$ conductivity contribution as becomes apparent upon breaking up the total conductivity into its individual contributions associated with $\mathrm{c}-\mathrm{c}, \mathrm{v}-\mathrm{c}$, and $\mathrm{v}-\mathrm{v}$ transitions according to Eq. (3). While the c-c contribution dominates the total conductivity at energies below $225 \mathrm{eV}$, the v-c contribution shows a pronounced threshold behavior at about $175 \mathrm{eV}$ and starts to prevail at energies above $225 \mathrm{eV}$. At the same time, the v-v transition contribution is almost negligible and can be associated with hopping processes. These can occur as a result of the partial filling of the $1 s$ states at the extreme densities and temperatures investigated here. This behavior is contrary to the known $0 \mathrm{~K}$ concept for solids, where the $\mathrm{v}-\mathrm{v}$ conductivity has to be zero, since the full occupation of the $1 s$ states leads to a vanishing transition probability due to selection rules.

Evaluation of the TRK sum rule for the total conductivity according to Eq. (2) yields a value of 5.91, which agrees with the exact sum rule value of 6 within $2 \%$. The same procedure applied to the $\mathrm{v}$-c contribution results in a value of 1.23 , which makes up about $21 \%$ of the total sum rule at these conditions. Additionally, the sum rule for the v-v transitions yields a value less than 0.01 , which translates to about $0.2 \%$ of the total value, and contributes the most at low energies. Finally, the largest total sum rule contribution of the remaining $79 \%$ is attributed to the sum rule value applied to the c-c conductivity contribution. The resulting c-c value is 4.67 and will be identified as the ionization state later in this work. Note that all sum rule values given in the following sections are 

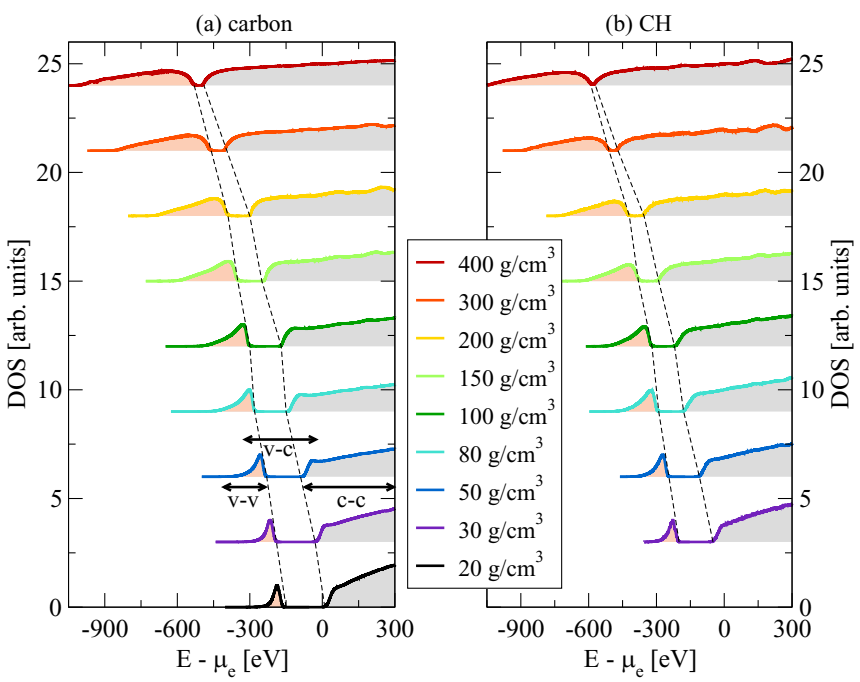

FIG. 2. Density of states of (a) carbon and (b) $\mathrm{CH}$ for different densities at $T=100 \mathrm{eV}$. The pink areas indicate states in the $1 \mathrm{~s}$ valence band, while states in the conduction band are colored in grey. The black arrows show exemplarily at $50 \mathrm{~g} / \mathrm{cm}^{3}$ the regions associated with intraband $(\mathrm{v}-\mathrm{v}, \mathrm{c}-\mathrm{c})$ and interband $(\mathrm{v}-\mathrm{c})$ transitions. The dashed lines serve as a guide to the eye for the valence-conduction gap.

corrected by a factor $Z^{\text {exact }} / Z^{\text {tot }}$ that accounts for the numerical uncertainty.

\section{B. Density of states}

In Fig. 2, we show our results for the DOS for carbon and $\mathrm{CH}$ for all considered densities at $100 \mathrm{eV}$. Each DOS shows a pronounced valence band corresponding to the $1 s$ states at small energies and the continuum of conduction states at high energies. Note that all energies are plotted with respect to the chemical potential $\mu_{\mathrm{e}}$.

For both materials, we observe the valence bands to broaden and shift towards smaller energies with increasing density. At the same time, the edge of the conduction states moves as well towards smaller energies and the gap between valence and conduction bands narrows with rising density. However, the gap never completely vanishes for the considered conditions and can be still clearly identified at the highest considered density of $400 \mathrm{~g} / \mathrm{cm}^{3}$.

The center of the gap between valence and conduction bands serves as input for our method to calculate the different conductivity contributions. In principle, any energy in the gap can be used to separate valence from conduction states. In this work, the gap was determined for every snapshot individually via the smallest energy difference between a state in the valence band and one in the conduction state. This approach is formally equivalent to the highest occupied molecular orbitallowest unoccupied molecular orbital method, which is applied at $T=0 \mathrm{~K}$ to obtain the energy difference between highest occupied and lowest unoccupied molecular orbital.

\section{Conductivity-based ionization}

In Fig. 3 we present our DFT-MD results for the ionization state of dense carbon for a temperature of $100 \mathrm{eV}$ as a function

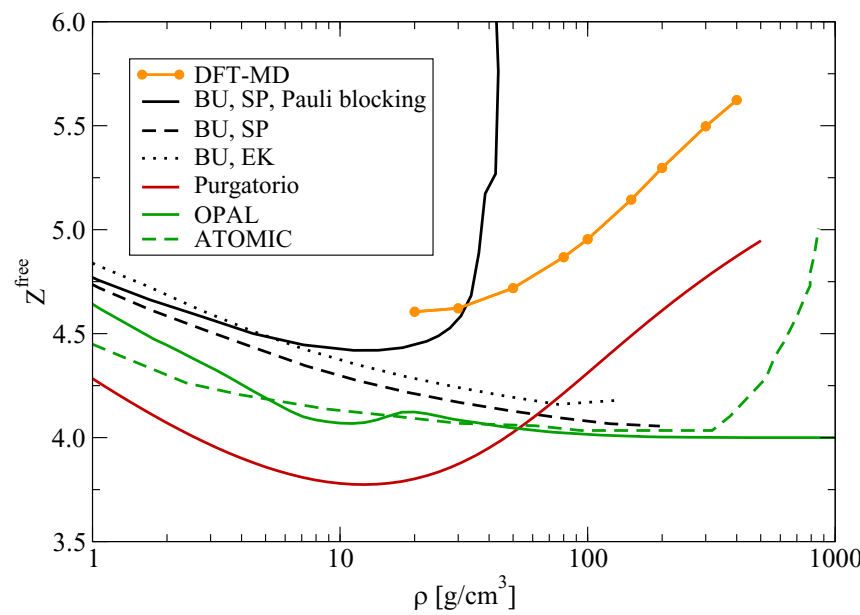

FIG. 3. Ionization state of carbon, $Z^{\text {free }}$, derived from DFT-MD simulations (orange line) according to Eq. (5) compared to predictions of PURGATORIO (red line), OPAL (green solid line) [5], ATMOIC (green dashed line) [45], and Beth-Uhlenbeck (BU) calculations (black lines) [21]. The BU results incorporate the two different IPD models by Ecker and Kröll (EK) and Stewart and Pyatt (SP), respectively, and the solid line additionally takes into account Pauli blocking effects.

of density compared to ionization models that are commonly used for modeling astrophysical or inertial confinement fusion (ICF) capsule implosions.

For the lowest densities considered here, the predictions for the carbon ionization state of PURGATORIO [46], ATOMIC [45], OPAL [5], and the different Beth-Uhlenbeck (BU) models [21] agree qualitatively and predict a decreasing ionization state with increasing density. However, two classes of models can be identified for the high-density regime. On one hand, OPAL and both BU models without Pauli blocking generally continue that trend at densities above $10 \mathrm{~g} / \mathrm{cm}^{3}$. Among the three models, OPAL predicts the smallest ionization state and converges towards a constant value of 4 . The BU curve including IPD based on the SP model gives slightly higher ionization states, but leads to no essential change in the general behavior compared to the BU approach using the EK description instead. On the other hand, the second class of curves, namely, PURGATORIO, ATOMIC, the BU model including Pauli blocking, as well as our DFT-MD results, is characterized by a steep rise at high densities, which is associated with pressure ionization expected under those conditions [47]. However, the slope and onset of this rise in ionization state vary vastly depending on the approach. The steepest slopes are predicted by ATOMIC and the BU model including Pauli blocking, which in contrast to the other two BU models includes electron degeneracy. While ATMOIC suggests the onset of the increase at about 300 $\mathrm{g} / \mathrm{cm}^{3}$, the BU model including Pauli blocking predicts this effect at a density an order of magnitude lower. The DFT-MD results confirm the increase, but the slope of our DFT-MD curve is not as steep. Furthermore, our calculations capture the slope of the average atom model PURGATORIO, which evaluates the effective charge at the Wigner-Seitz radius [46]. However, our $a b$ initio calculations yield systematically about 0.5 higher ionization states than PURGATORIO, indicating that 


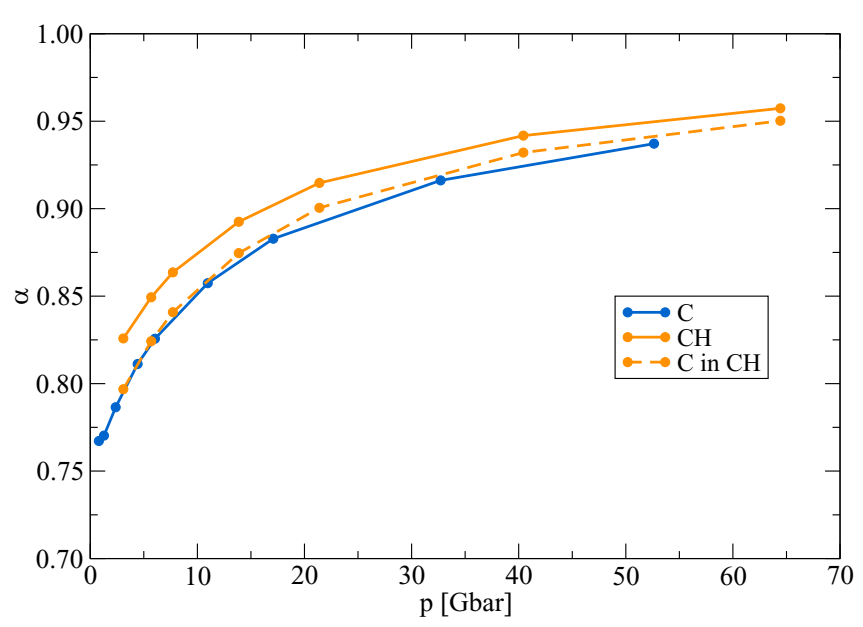

FIG. 4. Ionization degree $\alpha$ of pure carbon (blue line), $\mathrm{CH}$ (solid orange line), and carbon in $\mathrm{CH}$ assuming hydrogen to be fully ionized (dashed orange line).

this effective one-particle model is not capturing all important correlation effects treated via our many-body method.

The ionization degree derived according to Eq. (6) is shown as a function of pressure for carbon and $\mathrm{CH}$ as solid lines in Fig. 4. For both materials, we find a steady increase of the ionization degree in the range of 0.76 to 0.96 . Both curves have a very similar slope, while $\mathrm{CH}$ yields slightly higher ionization degrees than carbon. Additionally, we plot the ionization degree of the carbon in $\mathrm{CH}$ as a dashed curve, which agrees remarkably well with the values found for pure carbon indicating that the ionization degree of carbon is not changed by adding hydrogen. Note that this curve was calculated under the assumption that all hydrogen atoms are fully ionized. This assumption was tested for pure hydrogen at $80 \mathrm{~g} / \mathrm{cm}^{3}$, where we find an ionization degree of 1.00 as a result of the conductivity showing only a c-c contribution and of the vanished gap in the DOS.

\section{CONCLUSION}

Our presented method to derive the ionization state and ionization degree entirely from $a b$ initio simulations by applying the exact TRK sum rule for the dynamic electrical conductivity is entirely self-consistent. This approach naturally takes into account the electronic and ionic correlations; in particular, it reflects essential features of high-density plasmas such as the existence of energy bands instead of sharp atomic levels and their occupation according to Fermi-Dirac statistics. Our DFT-MD results for the carbon ionization state predict a gradually increasing pressure ionization and strongly disagree with commonly used models such as BU [21], OPAL [5], and ATOMIC [45] in high-density plasmas, yet we confirm the slope of the average atom model PURGATORIO [46]. This indicates that the degeneracy and many-body effects contained in our DFT-MD treatment are crucial to incorporate in any description of the ionization degree and that assumptions based on atomic physics are not valid to treat the ionization balance in high-density plasmas properly. Our conductivitybased method directly exploits knowledge of possible electronic transitions taking into account the nature of the wave functions of different states, which cannot be captured by a method that solely relies on the evaluation of the density of states, and additionally provides an experimentally accessible quantity. Hence, the presented data will be useful for analyzing and predicting conditions in inertial confinement fusion experiments using, e.g., the National Ignition Facility. In particular, these data will guide the understanding of XRTS spectra (see Refs. [48,49]).

Finally, the conditions considered in this work are typically found in high-density astrophysical objects. For instance, densities of $100 \mathrm{~g} / \mathrm{cm}^{3}$ and temperatures of $100 \mathrm{eV}$ are expected in the interior of $\mathrm{M}$ dwarf stars with a mass of $0.1 M_{\odot}$ (in units of the solar mass). Our results for the ionization degree of carbon and $\mathrm{CH}$ can be directly used as input for interior structure models, whose underlying radiation transport models and the nuclear reaction rates crucially rely on ionization models and opacities.

\section{ACKNOWLEDGMENTS}

We thank Luke B. Fletcher, Martin French, Dirk O. Gericke, Clemens Kellermann, Laurent Masse, and Martin Preising for helpful discussions. M.B., B.B.L.W., M.S., and R.R. acknowledge support by the Deutsche Forschungsgemeinschaft (DFG) within the FOR 2440. B.B.L.W., M.S., and S.H.G. were supported by the DOE Office of Science, Fusion Energy Science under FWP 100182. The work of T.D. and P.A.S. was performed under the auspices of the U.S. Department of Energy by Lawrence Livermore National Laboratory under Contract No. DE-AC52-07NA27344 and supported by Laboratory Directed Research and Development (LDRD) Grant No. 18-ERD-033. D.K. was supported by the Helmholtz Association under VH-NG-1141. The DFT-MD calculations were performed at the North-German Supercomputing Alliance (HLRN) facilities and the computing cluster Titan hosted at the ITMZ at University of Rostock.
[1] G. Chabrier and I. Baraffe, Structure and evolution of low-mass stars, Astron. Astrophys. 327, 1039 (1997).

[2] A. Becker, M. Bethkenhagen, C. Kellermann, J. Wicht, and R. Redmer, Material properties for the interiors of massive giant planets and brown dwarfs, Astron. J. 156, 149 (2018).

[3] R. Zimmermann, K. Kilimann, W.-D. Kraeft, D. Kremp, and G. Röpke, Dynamical screening and self-energy of excitons in the electron-hole plasma, Phys. Status Solidi B 90, 175 (1978).
[4] W.-D. Kraeft, D. Kremp, W. Ebeling, and G. Röpke, Quantum Statistics of Charged Particle Systems (Akademie-Verlag, Berlin, 1986).

[5] F. J. Rogers, F. J. Swenson, and C. A. Iglesias, OPAL equationof-state tables for astrophysical applications, Astrophys. J. 456, 902 (1996).

[6] M. S. Murillo and J. C. Weisheit, Dense plasmas, screened interactions, and atomic ionization, Phys. Rep. 302, 1 (1998). 
[7] B. J. B. Crowley, Continuum lowering-a new perspective, High Energy Density Phys. 13, 84 (2014).

[8] G. Ecker and Kröll, Lowering of the ionization energy for a plasma in thermodynamic equilibrium, Phys. Fluids 6, 62 (1963).

[9] J. C. Stewart and K. D. Pyatt, Jr., Lowering of ionization potentials in plasmas, Astrophys. J. 144, 1203 (1966).

[10] S. M. Vinko, O. Ciricosta, B. I. Cho, K. Engelhorn, H.-K. Chung, C. R. D. Brown, T. Burian, J. Chalupsky, R. Falcone, C. Graves, V. Hajkova, A. Higginbotham, L. Juha, J. Krzywinski, H. J. Lee, M. Messerschmidt, C. D. Murphy, Y. Ping, A. Scherz, W. Schlotter, S. Toleikis, J. Turner, L. Vysin, T. Wang, B. Wu, U. Zastrau, D. Zhu, R. W. Lee, P. A. Heimann, B. Nagler, and J. S. Wark, Creation and diagnosis of a solid-density plasma with an X-ray free electron laser, Nature 482, 59 (2012).

[11] O. Ciricosta, S. M. Vinko, H.-K. Chung, B.-I. Cho, C. R. D. Brown, T. Burian, J. Chalupsky, K. Engelhorn, R. W. Falcone, C. Graves, V. Hajkova, A. Higginbotham, L. Juja, J. Krzywinski, H. J. Lee, M. Messerschmidt, C. D. Murphy, Y. Ping, D. S. Rackstraw, A. Scherz, W. Schlotter, S. Toleikis, J. J. Turner, L. Vysin, T. Wang, B. Wu, U. Zastrau, D. Zhu, R. W. Lee, P. Heimann, B. Nagler, and J. S. Wark, Direct Measurements of the Ionization Potential Depression in a Dense Plama, Phys. Rev. Lett. 109, 065002 (2012).

[12] B. I. Cho, K. Engelhorn, S. M. Vinko, H.-K. Chung, O. Ciricosta, D. S. Rackstraw, R. W. Falcone, C. R. D. Brown, T. Burian, J. Chalupský, C. Graves, V. Hájková, A. Higginbotham, L. Juha, J. Krzywinski, H. J. Lee, M. Messersmidt, C. Murphy, Y. Ping, N. Rohringer, A. Scherz, W. Schlotter, S. Toleikis, J. J. Turner, L. Vysin, T. Wang, B. Wu, U. Zastrau, D. Zhu, R. W. Lee, B. Nagler, J. S. Wark, and P. A. Heimann, Resonant $K \alpha$ Spectroscopy of Solid-Density Aluminum Plasmas, Phys. Rev. Lett. 109, 245003 (2012).

[13] D. J. Hoarty, P. Allan, S. F. James, C. R. D. Brown, L. M. R. Hobbs, M. P. Hill, J. W. O. Harris, J. Morton, M. G. Brookes, R. Shepherd, J. Dunn, H. Chen, E. Von Marley, P. Beiersdorfer, H.-K. Chung, R. W. Lee, G. Brown, and J. Emig, Observations of the Effect of Ionization-Potential Depression in Hot Dense Plasma, Phys. Rev. Lett. 110, 265003 (2013).

[14] L. B. Fletcher, A. L. Kritcher, A. Pak, T. Ma, T. Döppner, C. Fortmann, L. Divol, O. S. Jones, O. L. Landen, H. A. Scott, J. Vorberger, D. A. Chapman, D. O. Gericke, B. A. Mattern, G. T. Seidler, G. Gregori, R. W. Falcone, and S. H. Glenzer, Observations of Continuum Depression in Warm Dense Matter with X-Ray Thomson Scattering, Phys. Rev. Lett. 112, 145004 (2014).

[15] D. Kraus, D. A. Chapman, A. L. Kritcher, R. A. Baggott, B. Bachmann, G. W. Collins, S. H. Glenzer, J. A. Hawreliak, D. H. Kalantar, O. L. Landen, T. Ma, S. LePape, J. Nilsen, D. C. Swift, P. Neumayer, R. W. Falcone, D. O. Gericke, and T. Döppner, $\mathrm{X}$-ray scattering measurements on imploding $\mathrm{CH}$ spheres at the National Ignition Facility, Phys. Rev. E 94, 011202(R) (2016).

[16] D. Kraus, B. Bachmann, B. Barbrel, R. W. Falcone, L. B. Fletcher, S. Frydrych, E. J. Gamboa, M. Gauthier, D. O. Gericke, S. H. Glenzer, S. Göde, E. Granados, N. J. Hartley, J. Helfrich, H. J. Lee, B. Nagler, A. Ravasio, W. Schumaker, J. Vorberger, and T. Döppner, Characterizing the ionization potential depression in dense carbon plasmas with high-precision spectrally resolved x-ray scattering, Plasma Phys. Controlled Fusion 61, 014015 (2018).
[17] M. F. Kasim, J. S. Wark, and S. M. Vinko, Validating continuum lowering models via multi-wavelength measurements of integrated x-ray emission, Sci. Rep. 8, 6276 (2018).

[18] S.-K. Son, R. Thiele, Z. Jurek, B. Ziaja, and R. Santra, Quantum-Mechanical Calculation of Ionization-Potential Lowering in Dense Plasmas, Phys. Rev. X 4, 031004 (2014).

[19] F. B. Rosmej, Ionization potential depression in an atomicsolid-plasma picture, J. Phys. B: At. Mol. Opt. Phys. 51, 09LT01 (2018).

[20] C. L. Lin, G. Röpke, H. Reinholz, and W. D. Kraeft, Ionization potential depression and optical spectra in a Debye plasma model, Contrib. Plasma Phys. 57, 518 (2017).

[21] G. Röpke, D. Blaschke, T. Döppner, C. Lin, W. D. Kraeft, R. Redmer, and H. Reinholz, Ionization potential depression and Pauli blocking in degenerate plasmas at extreme densities, Phys. Rev. E 99, 033201 (2019).

[22] S. M. Vinko, O. Ciricosta, and J. S. Wark, Density functional theory calculations of continuum lowering in strongly coupled plasmas, Nat. Commun. 5, 3533 (2014).

[23] S. X. Hu, Continuum Lowering and Fermi-Surface Rising in Strongly Coupled and Degenerate Plasmas, Phys. Rev. Lett. 119, 065001 (2017).

[24] K. P. Driver, F. Soubiran, and B. Militzer, Path integral Monte Carlo simulations of warm dense aluminum, Phys. Rev. E 97, 063207 (2018).

[25] C. A. Iglesias, A plea for a reexamination of ionization potential depression measurements, High Energy Density Phys. 12, 5 (2014).

[26] Carlos A. Iglesias and Philip A. Sterne, Comment on "Continuum Lowering and Fermi-Surface Rising in Strongly Coupled and Degenerate Plasmas", Phys. Rev. Lett. 120, 119501 (2018).

[27] S. X. Hu, Reply, Phys. Rev. Lett. 120, 119502 (2018).

[28] S. H. Glenzer et al., Cryogenic thermonuclear fuel implosions on the National Ignition Facility, Phys. Plasmas 19, 056318 (2012).

[29] R. Kubo, Statistical-mechanical theory of irreversible processes. I. General theory and simple applications to magnetic and conduction problems, J. Phys. Soc. Jpn. 12, 570 (1957).

[30] D. A. Greenwood, The Boltzmann equation in the theory of electrical conduction in metals, Proc. Phys. Soc. 71, 585 (1958).

[31] V. Recoules and J.-P. Crocombette, Ab initio determination of electrical and thermal conductivity of liquid aluminum, Phys. Rev. B 72, 104202 (2005).

[32] B. Holst, M. French, and R. Redmer, Electronic transport coefficients from ab initio simulations and application to dense liquid hydrogen, Phys. Rev. B 83, 235120 (2011).

[33] W. Thomas, Über die Zahl der Dispersionselektronen, die einem stationären Zustande zugeordnet sind. (Vorläufige Mitteilung), Naturwissenschaften 13, 627 (1925).

[34] F. Reiche and W. Thomas, Über die Zahl der Dispersionselektronen, die einem stationären Zustand zugeordnet sind, Z. Phys. 34, 510 (1925).

[35] W. Kuhn, Über die Gesamtstärke der von einem Zustande ausgehenden Absorptionslinien, Z. Phys. 33, 408 (1925).

[36] G. D. Mahan, Many-Particle Physics (Plenum Publishers, New York, 2000). 
[37] M. P. Desjarlais, J. D. Kress, and L. A. Collins, Electrical conductivity for warm, dense aluminum plasmas and liquids, Phys. Rev. E 66, 025401(R) (2002).

[38] G. Kresse and J. Hafner, Ab initio molecular-dynamics for liquid-metals, Phys. Rev. B 47, 558 (1993).

[39] G. Kresse and J. Hafner, Ab initio molecular-dynamics simulation of the liquid-metal-amorphous-semiconductor transition in germanium, Phys. Rev. B 49, 14251 (1994).

[40] G. Kresse and J. Furthmüller, Efficient iterative schemes for $a b$ initio total-energy calculations using a plane-wave basis set, Phys. Rev. B 54, 11169 (1996).

[41] S. Nosé, A unified formulation of the constant temperature molecular dynamics methods, J. Chem. Phys. 81, 511 (1984).

[42] A. Baldereschi, Mean-value point in the Brillouin zone, Phys. Rev. B 7, 5212 (1973).

[43] J. P. Perdew, K. Burke, and M. Ernzerhof, Generalized Gradient Approximation Made Simple, Phys. Rev. Lett. 77, 3865 (1996).

[44] J. Sun, A. Ruzsinszky, and J. P. Perdew, Strongly Constrained and Appropriately Normed Semilocal Density Functional, Phys. Rev. Lett. 115, 036402 (2015).
[45] P. Hakel, M. E. Sherill, S. Mazevet, J. Abdallah, Jr., J. anf Colgan, D. P. Kilcrease, N. H. Magee, C. J. Fontes, and H. L. Zhang, The new Los Alamos opacity code ATOMIC, J. Quantum Spectrosc. Radiat. Transf. 99, 265 (2006).

[46] P. A. Sterne, S. B. Hansen, B. G. Wilson, and W. A. Isaacs, Equation of state, occupation probabilities and conductivities in the average atom Purgatorio code, High Energy Density Phys. 3, 278 (2007).

[47] N. F. Mott, The transition to the metallic state, Philos. Mag. 6, 287 (1961).

[48] B. B. L. Witte, L. B. Fletcher, E. Galtier, E. Gamboa, H. J. Lee, U. Zastrau, R. Redmer, S. H. Glenzer, and P. Sperling, Warm Dense Matter Demonstrating Non-Drude Conductivity from Observations of Nonlinear Plasmon Damping, Phys. Rev. Lett. 118, 225001 (2017).

[49] D. Kraus, T. Döppner, A. L. Kritcher, A. Yi, K. Boehm, B. Bachmann, L. Divol, L. B. Fletcher, S. H. Glenzer, O. L. Landen, N. Masters, A. M. Saunders, C. Weber, R. W. Falcone, and $\mathrm{P}$. Neumayer, Platform for spectrally resolved X-ray scattering from imploding capsules at the National Ignition Facility, J. Phys. Conf. Ser. 717, 012067 (2016). 